\title{
SCALE-INVARIANT AND WAVE NATURE OF THE HUBBLE PARAMETER
}

\author{
Zhanabaev Z.Zh., Ussipov N.M. ${ }^{\star}$, Khokhlov S.A. \\ Al-Farabi Kazakh National University, Almaty, Kazakhstan, ussipov.nurzhan@kaznu.kz
}

\begin{abstract}
The value of the global Hubble parameter corresponding to astrophysical observations was determined theoretically without using ${ }_{\wedge}$ CDM models. A nonlinear fractal model of the connection between the distance to the observed galaxy and its coordinate is proposed. Distance is defined as a fractal measure, the measurement scale of which, in contrast to the known fractal models, corresponds to the deviation of the desired measure itself from its fixed value (radius of zero gravity), relative to which the scale invariance is assumed. We used the dimension of our proposed specific anisotropic fractal, which simulates the increase in the distance to the observation point. It is shown that this dimension is also the maximum dimension of the strange attractor of the phase portrait of the equation of gravitational waves and sets of galaxies from different catalogs.
\end{abstract}

Keywords:Hubble parameter, gravitational waves, fractal dimension, expansion of the universe.

\section{Introduction}

The works [1-3] present the results of determining the rate of expansion of the universe. At distances of at least 3 million light-years (Mpc), galaxies can separate at a speedof $68-73 \mathrm{~km} / \mathrm{s}$. The discrepancy between the results and their average is about $4 \%$.

To describe the observed regularity, the expansion of the universe along with $\Lambda C D M$ model, the cosmological constant $\Lambda$ is added to the Einstein gravitational field equation, the need to comprehend which led to the unclear concept of dark energy. Recently, modified theories of gravity have been put forward as an alternative to the dark energy hypothesis [4]. These theories are aimed at finding more complex combinations of multidimensional space curvature invariants. It is expected that the modified theory of gravity may be applicable to high energy physics problems. However, the presence of higher derivatives with new additional parameters leads to new problems in the quantitative description of the observation results. Multidimensionality, the need for additional measurements are indicated in contemporary studies on the studied issue. One of the possibilities to take into account additional dimensions is to take into account the fractional dimension of fractal objects, which are scale-invariant and self-similar in structure.

In works [5-7], an alternative possibility to $\Lambda$ CDM model called the Scale Invariant Vacuum (SIV) theory, where the cosmological constant is multiplicatively related to the theoretically introduced coefficient of scale invariance of space, is developed. Several tests of the SIV results were carried out with cosmological observations of the Hubble parameter, redshift, and its connection with temperature. Good agreement between the results of theory and observations was obtained. It is concluded that observational facts can be described without using hypotheses about dark energy and dark matter.

The scale invariance of geometric objects manifests itself in the form of fractal structures: a small part repeats the shape of the whole. Since the works of B. Mandelbrot, the theory of fractals has been widely used in astrophysics. However, the inconsistency of the theory with some observational facts, for example, with spectral laws, makes it necessary to use "new types of fractals" [8]. We will consider the possibilities of using new models of fractal sets: describing the formation of pre-fractals in only one direction and a nonlinear fractal measure (distance to galaxies), the measurement scale of which depends on the measure itself.

The aim of this work is to determine the value of its fractal dimensions from the attractor obtained from the equation of weak gravitational waves and use them in the equation for the distance to galaxies to determine the global value of the Hubble parameter without using the cosmological constant and to compare the results of theory with observations. 


\section{Scale invariance of the attractor of signals of gravitational waves}

Einstein's equations for the gravitational field have the form

$$
R_{i j}=8 \pi G\left(T_{i j}-\frac{1}{2} g_{i j} T\right)
$$

where $R_{i j}$ is the space curvature tensor (Ricci tensor), $T_{i j}$ is the energy-momentum tensor, $g_{i j}$ is the metric tensor, $G$ is the gravitational constant, and the speed of light $c=1$ is taken.

Let us assume that inhomogeneities in the distribution of matter cause inhomogeneities in the geometry of space. Then, in a synchronous frame of reference, the elements of the Galilean metric tensor with small perturbations $\left|h_{\alpha \beta}(R, t)\right| \ll 1$ can be written in the form [8, 9]:

$$
g_{00}=1, \quad g_{0 \alpha}=0, \quad g_{\alpha \beta}=-a^{2}(t)\left[\delta_{\alpha \beta}-h_{\alpha \beta}(R, t)\right],
$$

where $a(t)$ is the scale factor of the expansion of the universe, $h_{\alpha \beta}(R, t)$ are linear perturbations of the curvature of space, and $\alpha, \beta$ are the components of the spatial variable $X$. obtained

Taking into account conditions (2) from equation (1) in [8] the equation of gravitational waves is

$$
a^{-2} \nabla^{2} h_{\alpha \beta}=\ddot{h}_{\alpha \beta}+3\left(\frac{\dot{a}}{a}\right) \dot{h}_{\alpha \beta},
$$

where $\nabla$ are spatial derivatives, a dot means time derivative.

For the Fourier components with a spatial wavenumber $k$ associated with the length of the transverse gravitational waves $\lambda=2 \pi a(t) / k$, we have the equation for the perturbations of single polarization $h(t)$ :

$$
\frac{d^{2} h(R, t)}{d t^{2}}+3\left(\frac{\dot{a}}{a}\right) \frac{d h(R, t)}{d t}+\left(\frac{k}{a}\right)^{2} h(R, t)=0 .
$$

The last term in (4) has the dimension $1 / \mathrm{m}^{2}$, and the first two $-1 / \mathrm{s}^{2}$, because it was assumed that the speed of light is equal to unity. Let us come in (4) to the spatial derivatives by $R: \frac{\partial}{\partial t} \rightarrow v_{0} \frac{\partial}{\partial R}$, it is assumed that $v_{0}=1$ is the velocity of gravitational waves in a medium with cosmic perturbations of the density of fields and matter:

$$
\ddot{h}+3\left(\frac{\dot{a}}{a}\right) \dot{h}+\left(\frac{k}{a}\right)^{2} h=0,
$$

where dots denote derivatives to the coordinate $R$.

The derivation of equation (5) is given in [8]. The choice of this form from the set of equations of gravitational waves [9] can be justified only within the framework of the same used approximations, neglecting the square of small quantities. Equation (5) describes the wave process of change $h(R)$ at a fixed time. By definition $\frac{k}{a}=\frac{2 \pi}{\lambda}$ is the ratio of the wavenumber to the scale factor. To clarify the meaning of $3 \frac{\dot{a}}{a}$, we assume that the mass of matter $M$ with its density $\rho$, with the scale factor of expansion of the volume $a$,and also with perturbations $d \rho$, daare related as

$$
\frac{3}{4 \pi} M=\rho a^{3}, \frac{3}{4 \pi} M=(\rho-d \rho)(a+d a)^{3} .
$$

Dividing these equations term by term, neglecting the squares and cubes of small quantities $d \rho, d a$, through the derivatives $\dot{\rho}=d \rho / d R, \dot{\rho}=d \rho / d R$, we obtain

$$
-\frac{\dot{\rho}}{\rho}=3 \frac{\dot{a}}{a} .
$$

For $\frac{\dot{\rho}}{\rho}>0$, we have damped oscillations; for $\frac{\dot{\rho}}{\rho}<0$ Eq. (5) describes undamped oscillations in R. 
Let us introduce the Hubble parameter $H=\dot{a} / a$ as a dynamic characteristic of the problem and write equation (5) in the form of a system convenient for numerical analysis:

$$
\dot{h}=b, \quad \dot{a}=H a \quad \dot{b}=-3 H b-\left(\frac{a}{R_{*}}\right)^{2} h,
$$

In contrast to equation (5), in the system of equations (8) the wavenumber $\mathrm{k}$ is represented through a physical characteristic, the radius of zero gravity $R_{*}$, since gravitational waves with a characteristic length $\lambda=2 \pi\left(R_{*} / a\right)$ can be realized in clusters of galaxies:

$$
\frac{k}{a}=\frac{2 \pi a}{\lambda a}=\frac{a}{R_{*}}
$$

Various value $R_{*}$ estimatesare accepted. More generally, $R_{*}$ values can be taken as the Lagrange points of interacting stars. On the scale of galaxies, zero-gravity points lead to fluctuations in the motion of galaxies. Before the establishment of global expansion in distance, both the repulsion and the attraction of galaxies take place, which is observed as fluctuations and instability of their coordinates. The transition to global expansion can be considered as a result of large-scale fluctuations in the form of a phase transition [10]. The stability of the Lagrange points of gravitating systems is analyzed in contemporary studies [11]. Due to the scale invariance of the studied phenomenon, regularities will be established for $R_{*}=1$, the values $R_{*}>1$ are needed to analyze the role of many galaxies with different $R_{*}$.

For short waves $\left(\frac{\lambda}{R_{*}} \sim \frac{a}{k R_{*}} \ll 1\right)$, from (5) a solution for $h(R)$ follows in the form of harmonic functions. The shape of long waves is affected by the inhomogeneity of the cosmic background (fluctuations in the density of matter, radiation).As an example the results of a numerical analysis of the system (8) with the initial values $h_{0}=0.01 ; b_{0}=0.01 ; a_{0}=1.01$ and parameters $R_{*}=10, H=10$ are shown in Figure 1 .

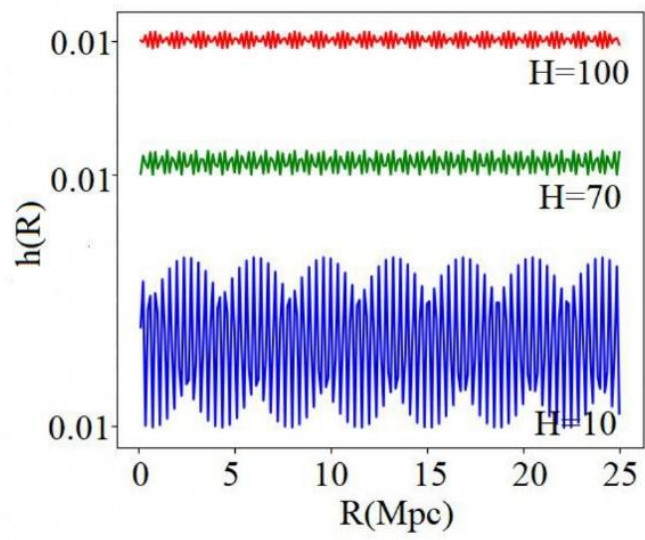

a)

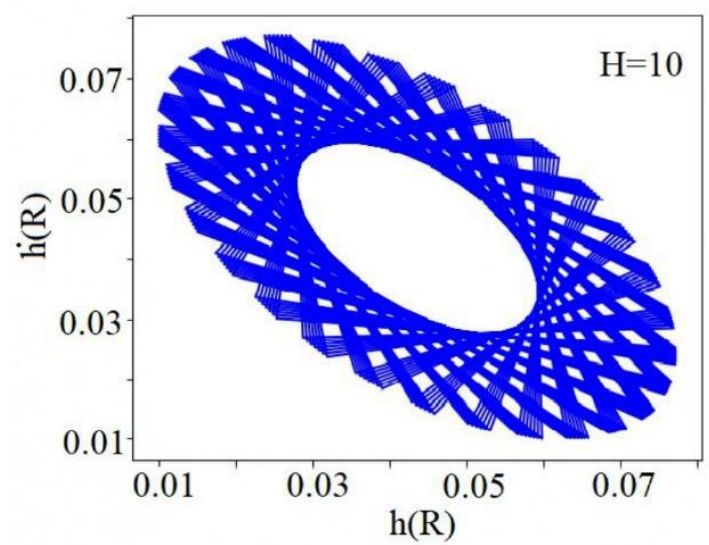

b)

Fig.1. Gravitational-wave shapes (a), and its basic portrait (b).

The values $h(R)$ decrease with increasing $H$ and the oscillations become structured chaotic.Dynamical system (8) has phase portraits $(h(R), \dot{h}(R))$ in the form of fractal sets for different values of the parameter $H$. The correlation dimensions of attractors $D$ were determined by the expression

$$
D=\lim _{\delta \rightarrow 0} \frac{\log C(\delta)}{\log \delta}, C(\delta)=\lim _{N \rightarrow \infty} \frac{1}{N^{2}} \sum_{i=1}^{N} \sum_{j \neq i}^{N} \theta\left(\delta-\left\lceil x_{i}-x_{j}\right\rceil\right),
$$

where $\delta$ is the measurement scale, $C(\delta)$ is the correlation integral, $N$ is the number of points in the set, $\left[x_{i}-x_{j}\right]$ is the distance between two pairs of points $(i \neq j), \theta(f)$ is the Heaviside function.

As the values $\delta$ increase, $C(\delta)$ saturate, the corresponding maximum value $D_{*}$ is observed in the interval $50 \leq H \leq 75$ (Fig. 2). 


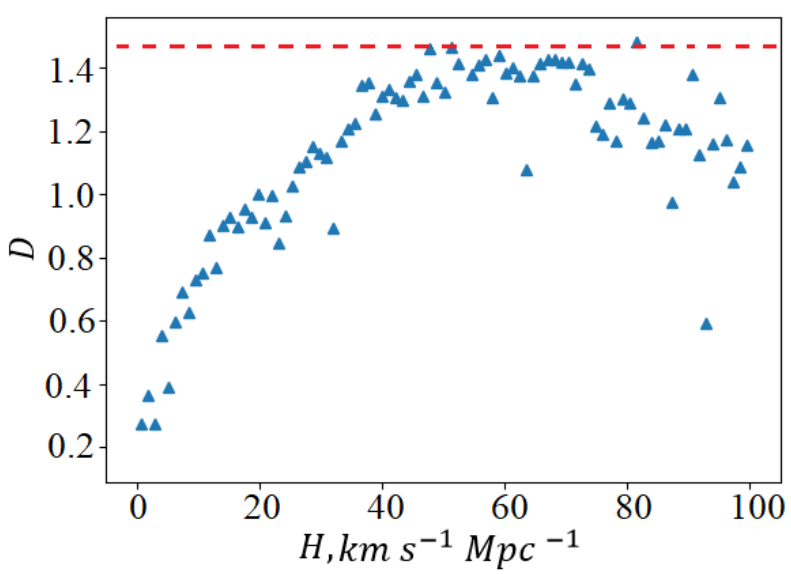

Fig.2. Possibility of maximum fractal dimension of a strange attractor of a dynamical system (8).

According to the theory, we found the value of $\mathrm{H}$ divided by the background velocity $v_{0}=1 \mathrm{kms}^{-1}$. The absolute value of the Hubble parameter $H$ is in $\mathrm{kms}^{-1} \mathrm{Mpc}^{-1}$ units. The exact value of the maximum $D=D_{*}=1.465$ corresponds to the theoretical model of the original fractal and the analysis of galaxy catalogs, which are given in Section 4.

\section{Fractal dimension of the sets of expanding galaxies}

There is a geometric model of a fractal with a value $\gamma_{*}=D-d=0.465$, where $d$ is the topological dimension, $D$ is the fractal dimension. This fractal (Fig. 3), called anisotropic, was proposed by one of the authors of this work to describe turbulence in the boundary layer and to create an information-efficient fractal antenna [12, 13]. Each pre-fractal (hierarchical levels of order $n$ ) has 5 links with a relative length of $1 / 3$. The vertical links are not pre-fractals of the next order.

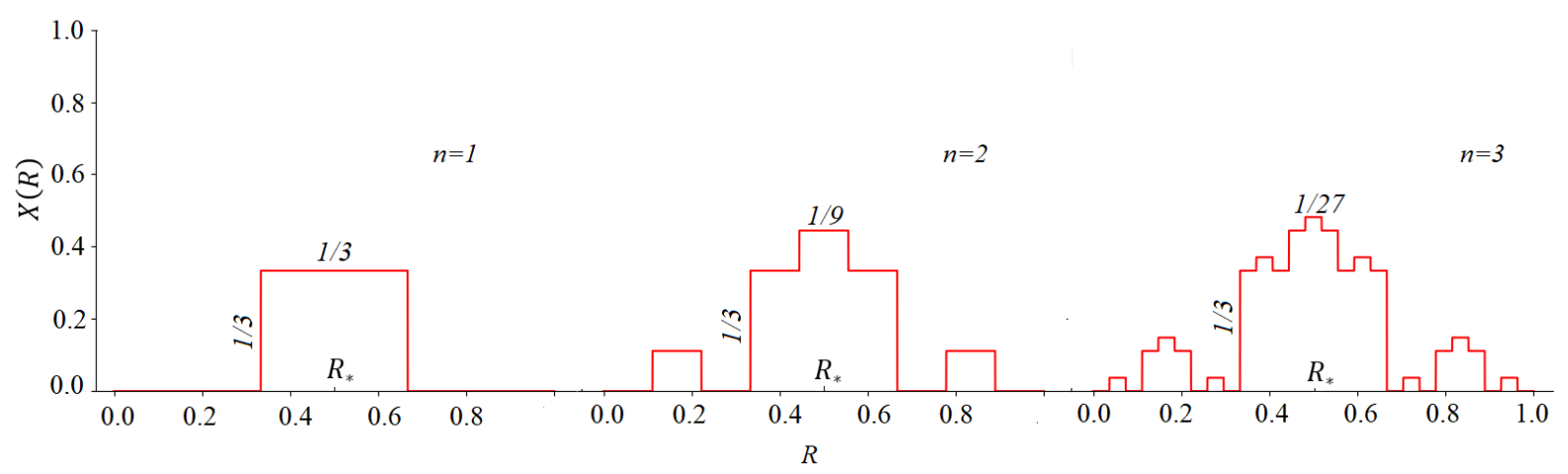

Fig.3. Anisotropic fractal with pre-fractals of order $n=1,2,3$.

The Hausdorff local dimension of all pre-fractals is of order $\mathrm{n}$ of an anisotropic fractal $D=D_{*}=$ $\ln N / \ln (1 / \delta)=\ln 5 / \ln 3=1.465$, where $\mathrm{N}$ is the number of links, $\delta$ is the dimensionless measurement scale. Other fractals are known [14] with the same dimension $D$, which does not depend on the direction of formation of pre-fractals, but their topology does not reflect the specifics of the one-sided wave expansion of galaxies. The point is that the dimension $D$ of the set of hierarchies of an anisotropic fractal manifests itself only in one direction (Fig. 3). For astrophysical applications, it can be denoted through a $R$ coordinate, through $X(R)$ - the distance to the galaxy.

\section{Nonlinear geometric model of the expansion of the universe}

Since the works of B. Mandelbrot, the theory of fractals has been used to describe the structure of the universe on a large scale. However, for a more detailed adequate description of cosmological observations, new concepts of fractal geometry are needed. The well-known theories of fractals imply an independent choice of the scale of measurement of a measure (additive geometric, physical quantity) from the value of 
the measure itself. Near critical values, for example, at $R \approx R_{*}$ the measure varies greatly, it is necessary to take into account the indicated dependence. Using the Hausdorff expression, we define the fractal length $X(R, \gamma)$ in the form

$$
X(R, \gamma)=R\left(\frac{\Delta X(R, \gamma)}{R_{*}}\right)^{d} \cdot\left(\frac{\Delta X(R, \gamma)}{R_{*}}\right)^{-D}=R\left(\left|1-\frac{X(R, \gamma)}{R_{*}}\right|\right)^{-\gamma},
$$

where $\quad \Delta X(R, \gamma)=\left|X(R, \gamma)-R_{*}\right|, \gamma=D-d$

The Eq. (11) must be analyzed taking into account the fractality of the desired length $X(R, \gamma)$, which is possible through discrete iteration over pointswith step $i$ as a display:

$$
X_{i+1}(R, \gamma)=R\left(\left|1-\frac{X_{i}(R, \gamma)}{R_{*}}\right|\right)^{-\gamma}
$$

The value of the indexi $i$ in the notation of a continuous function $X_{i+1}(R, \gamma)$ corresponds to the steady-state of the display sequence (12), regardless of the initial conditions. In terms of the cosmological equation [8] $X_{i+1}(R, \gamma)$ determines the possible distance to the galaxy with the coordinate $R$, the right side of Eq. (12) is the scale factor $a(t)$ multiplied by $R$ depending on the coordinate $X_{i}$. In what follows, we will use the notation $X(R, \gamma)$ omitting the index $i+1$. Changing the measure of space depends on itself. With an increase in the parameter $\gamma$, the values $X(R, \gamma)$ become chaotic near $R \approx R_{*}$ (Fig. 4$)$.

The results of iterating over the values $X(R, \gamma)$ in points will differ from the results of standard computer methods, where segment iteration is used. For clarity, Fig. 4 shows curves with a large step $\Delta R=10^{-1}$. When decreasing $\Delta R$ to $10^{-3}$, the smoothed character of curve 3, obtained using the Scipy package, in comparison with curve 2 , remains at the same number of samples.

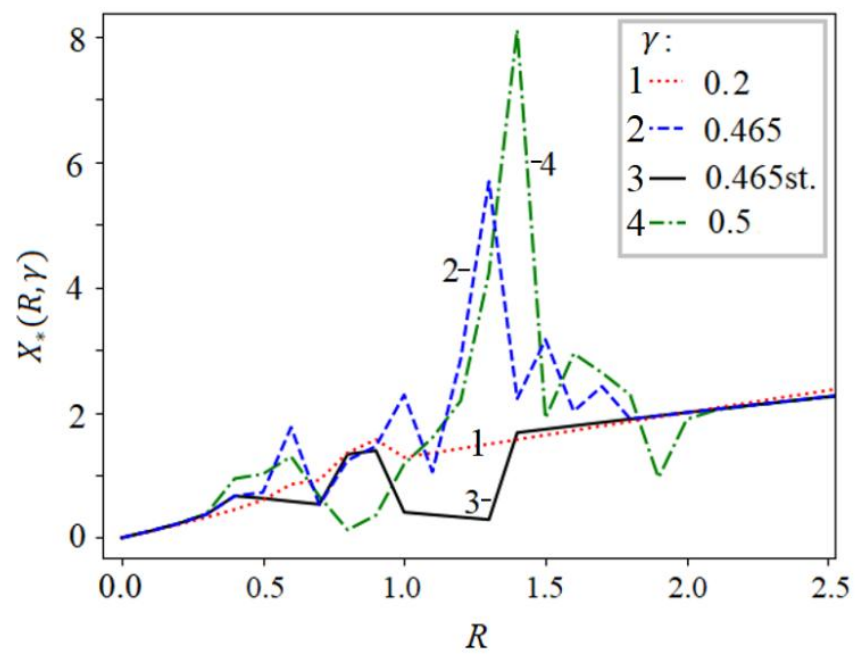

Fig.4.Chaotization of values $X(R, \gamma)$ with an increase in the scaling index $\gamma$ at $R_{*}=1$.

The number of display iterations (12) equals $10^{3}$.

If relative values of variables $X / R_{*} R / R_{*}$ are used in Eq. (12), the form of the equation does not change. Therefore, according to the definition of the Hubble parameter $\mathrm{H}$ for $R_{*}=1$, we have:

$$
\left.H=\frac{1}{X(R, \gamma)}(\Delta X(R, \gamma)) / \Delta R\right),
$$

where $\Delta X / \Delta R$ is the rate of removal of galaxies, along the coordinate $R \Delta X=X_{i+1}-X_{i}, \Delta R$ - coordinate change corresponding to $\Delta X$. Let us take the minimum value $\Delta R$ equal $\sigma_{X}$ - the standard deviation of $X(R, \gamma)$.

A stable value $\sigma_{X}=\sigma_{*}=0.0149$ at $\gamma<\gamma_{*}$, but $\gamma_{*}$ corresponds to the global expansion of the universe. Close values to $\sigma_{*}$ are also observed for some forms of singular generalized functions (like $\delta$ - the Dirac function) when analyzing their behavior by an iterative method near the point of a burst ("explosion"). 


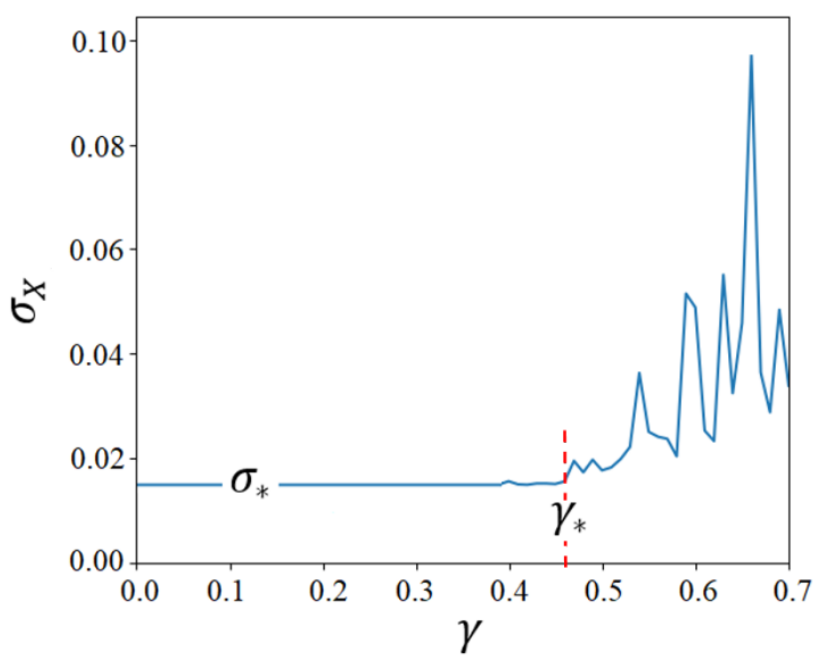

Fig.5. Change in standard deviation $X(R, \gamma)$ by $\gamma$ according to Eq. (12). $\sigma_{*}=0.0149, \gamma_{*}=0.465$

(by Figures 2, 3, and subsequent 6, 8).

It should be taken into account that the generalized functions expressed in terms of harmonic functions give a value of $\sigma_{x} \sim 0.18$ due to the well-known Gibbs phenomenon, which occurs when a jump function is approximated by a Fourier series. Possibilities of decreasing $\sigma_{x}$ in these cases are considered in contemporary studies [15]. Close values to $\sigma_{*}$ are observed for examples in the form of a power-law generalized function since the nonlinear fractal measure $X(R, \gamma)$ contains a degree $\gamma$. For example, for the function $f(x)=$ $\frac{M}{\sqrt{\pi}} e^{-(n x)^{2}}$, iterative analysis at $M \rightarrow \infty$ in a change range $n$ corresponding to the randomization of the value $x$ with a positive Lyapunov exponent gives the result $\sigma_{x}=0.018$.

The accuracy of determining the coordinates of galaxies $R$ and the parameter $H$ are interdependent. The root-mean-square deviations $\sigma_{R}, \sigma_{H}$ can be estimated from the condition of violation of the coherence of the chaotic characteristics of the gravitational wave near $R \approx R_{*}$ :

$$
\Delta \lambda \Delta k \geq 2 \pi
$$

where $\Delta \lambda, \Delta k$ are the moduli of wavelength $\lambda$ and wavenumber fluctuations $k$. Wavelength fluctuations are proportional to changes in the geometric characteristics - coordinates $R: \Delta \lambda \sim \Delta R$ Wavenumber fluctuations are inversely proportional to the change in the fractal distance $\Delta k \sim \Delta\left(\frac{1}{X(R, \gamma)}\right)$. By definition of the Hubble parameter, its change is proportional to $\Delta H \sim \Delta\left(\frac{1}{X(R, \gamma)}\right)$. Consequently, $\Delta k \sim \Delta H$. After that, relation (14) for the relative standard deviations has the form

$$
\sigma_{R} \sigma_{H} \geq 2 \pi,
$$

where $\sigma_{R}=\left((\Delta R)^{2}-\left(<\Delta R>^{2}\right)\right)^{\frac{1}{2}} / R_{*}, \sigma_{H}=\left((\Delta H)^{2}-\left(<\Delta H>^{2}\right)\right)^{1 / 2} / H_{\max }$

A theoretical estimate $H_{\max }=H\left(\gamma_{*}\right)$ is possible according to equation (5).

\section{Comparison of theoretical results with cosmological observations}

The values of the fractal dimension of the sets of galaxies determined from catalogs differ. For example, in [16], the values of fractal dimension $\mathrm{D}=1.3-1.5$ for the SDSS $16^{\text {th }}$ data release catalog were obtained by the correlation method.To increase the accuracy of determining $\mathrm{D}$, we calculated the correlation function in Eq. (10) for $r_{i j_{\text {min }}}<\delta<r_{i j_{\text {max }}}$, where $r_{i j_{\text {min }}}, r_{i j_{\text {max }}}$ are the smallest and largest distance between two points. The choice of $r_{i j}$ max is determined by the saturation of the number of pairs of points (Figure 6a.).

Indeed, the fractal dimension $D=1.465$ is installed only on a certain spatial scale (Fig. $7 \mathrm{~b}$ ). Many galaxies form a fractal correlation curve with the topological dimensiond $=1$. The maximum value of the scaling index corresponds to its theoretical value $\gamma_{*}=D-d=0.465$. 


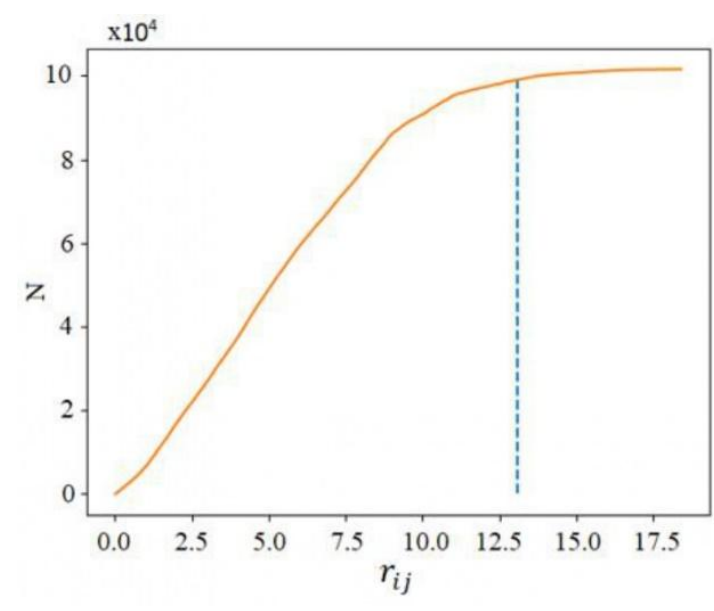

a)

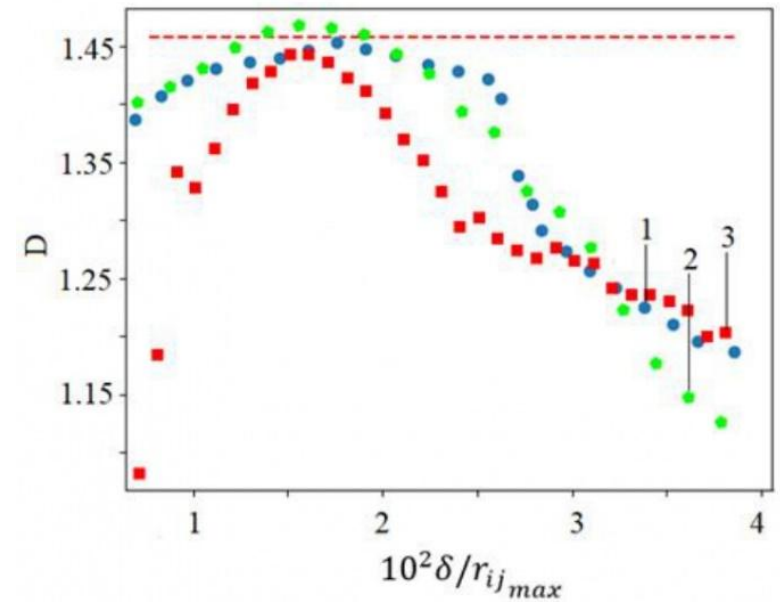

b)

Fig. 6.Saturation of the number of pairs of points $\mathrm{N}$ by the distance between two points according to the catalog [17] (a). Change in the correlation dimension according to the relative scale of measurement $10^{2} \delta / r_{i j}$ max according to catalogs: 1- [ 17], 2- [18], 3- [19](b).

Figure 7 shows a Hubble diagram for a set of values $10 \leq R_{*} \leq 200, \gamma=\gamma_{*}$. The theoretical values $H, \mathrm{kms}^{-1} \mathrm{Mpc}^{-1}=65,72,79$ were obtained for $\frac{\Delta R}{R_{*}} * 10^{2}=1.36 ; 1.49 ; 1.65$ by averaging $N=10^{4}$ points. There is no global expansion rate $\gamma<\gamma_{*}$ near the Local Group $(1 \leq R \leq 3 \mathrm{Mpc})$.

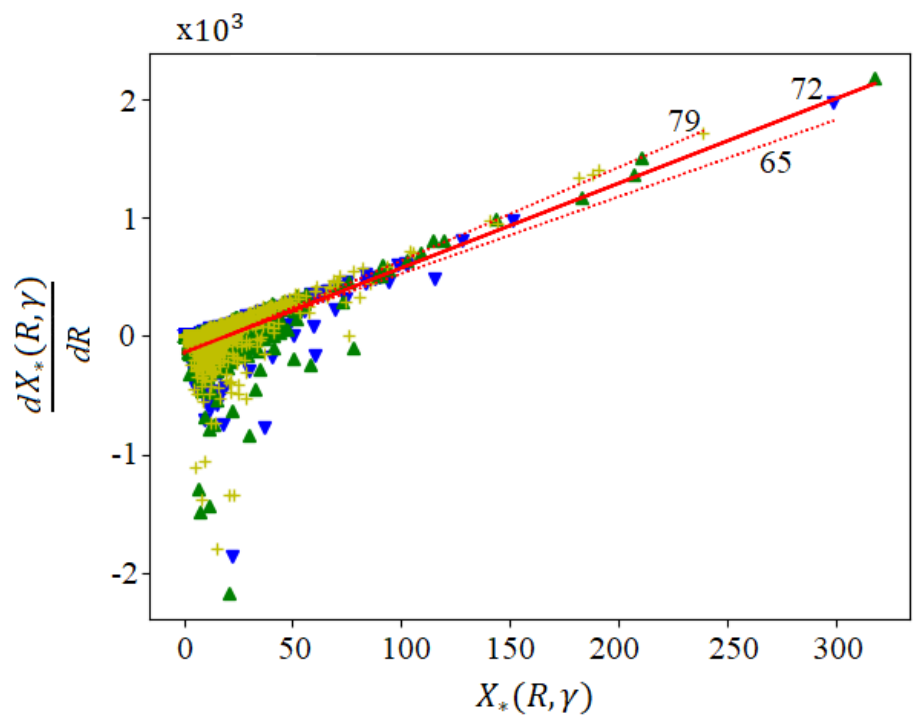

Fig.7. An illustration of the existence of the global value for the Hubble parameter for $\gamma=\gamma_{*}$. The average value of the interval $H=65-79$ (inunitskms ${ }^{-1} \mathrm{Mpc}^{-1}$ ) according to the results of observations [1-3, 20] is 72 with an error of about $4 \%$. The value $H$ according to the present theory at $\gamma=0.465, \sigma_{R}=0.0149$ is $72, \Delta H=2 \pi / 1.49=4.21 \%$.

The ratio of the speed of galaxies to the speed of the background $\frac{\Delta X(R, \gamma)}{\Delta R}$ varies along the coordinate $R$ and can be both positive and negative, as in astrophysical observations [1-3, 20]. The global expansion of the universe is described by the value $\gamma_{*}=0.465$. Saturation of $\mathrm{H}$ value by the increase of $\gamma$ to $\gamma_{*}$ determined by the analysis of the attractor of gravitational waves (Fig. 2) is also observed from the result of the theory of the distance to galaxies as a nonlinear fractal measure (Fig. 8). Objects of the same luminosity, located at different distances from the observer, will have different apparent values.

Observations [21] show that the luminosity (m-M) increases chaotically with increasing redshift $z$ and its behavior towards saturation can be described by the relation:

$$
m-M=5 *\left(\log _{10} L+5\right),
$$


where $L$ is the measured redshift distance to the galaxy.

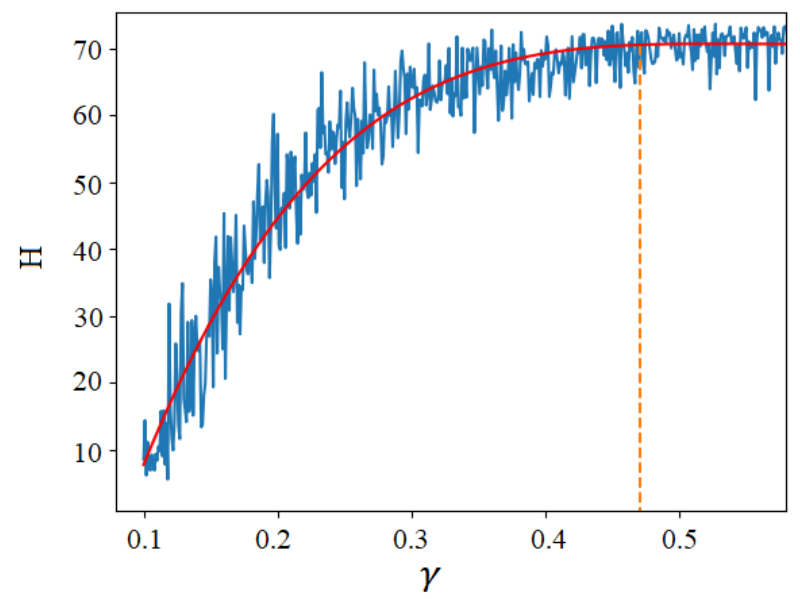

Fig.8.Changes in the Hubble parameter with increasing scaling exponent at $\Delta R=1.49 * 10^{-2}$.

To compare the result (16) with the present theory, we replace $\mathrm{L}$ by $X(R, \gamma)$ from Eq. (12). We define the redshift as the rate of change in the distance along the coordinate $z=\frac{\Delta X(R, \gamma)}{\Delta R}$, select the value $\Delta R=$ $1.58 * 10^{-2} \mathrm{Mpc}$ as corresponding to $H=68 \mathrm{kms}^{-1} \mathrm{Mpc}^{-1}$ that adopted in [21], and obtain chaotic values of the luminosity (Fig. 9). The regular curve in this figure, as in [21], corresponds to the values $L=z / H$

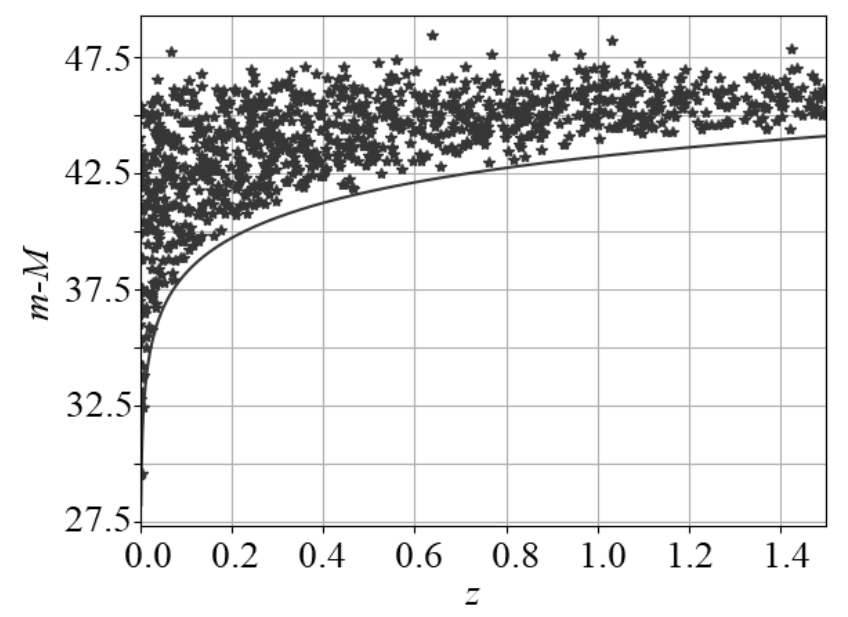

Fig.9. Change of luminosity value by redshift, corresponding to equations (16) and (12).

\section{Conclusion}

The equation for weak perturbations of the curvature of space, following from Einstein's equations for the gravitational field, is written in terms of the dependence of the wave number on the scale factor of the expansion of the universe and the radius of zero gravity of galaxy clusters $R_{*}$. The attractor of this equation has the maximum fractal dimension $D=d+\gamma_{*}=1+0.465$, which is reached when the Hubble parameter is changed. The value of the scaling exponent $\gamma_{*}$ corresponds to the new anisotropic fractal model, various applications of which were previously considered by the authors, and to the correlation fractal dimension of the distribution of galaxies in various catalogs.

The distance to the galaxy is determined through its coordinate as a nonlinear fractal measure, the measurement scale of which, in contrast to the known fractal models, takes into account the deviation of the desired measure itself from its value at the point of zero gravity $R_{*}$ (at the Lagrange points of gravitating objects). The change in the distance along the coordinate determines the ratio of the galaxy's velocity to the velocity of the system accompanying the gravitational wave and the corresponding Hubble parameter. 
Discrete iterative analysis of a singular nonlinear equation with a fractional power for distance gives a scale-invariant value of the root-mean-square deviation of 0.0149 for $R_{*}=1$. Such results are typical for examples of singular generalized functions obtained by discrete iterative analysis, taking into account the structure of the fractal measure. The use of the established minimum measurable interval of the galactic coordinate value is in good agreement with the latest observations of the Hubble parameter, redshift in distance, the dependence of luminosity on redshift. Due to the self-similar wave nature, the value of the Hubble parameter depends on the ratio of the violation of the wavelength coherence and the wavenumber of the gravitational wave. Thus, new models of fractal measures (objects of non-Euclidean geometry) make it possible to describe the basic observational facts of cosmology by Einstein's ideas about homogeneity and Mandelbrot's about the scale invariance of the universe.

\section{Acknowledgements \\ This research has been funded by the Science Committee of the Ministry of Education and Science of the Republic of Kazakhstan (Grant No. AP08856419).}

\section{REFERENCES}

1 Riess A. G. et al. A $2.4 \%$ determination of the local value of the Hubble constant. The Astrophysical Journal. 2016, Vol. 826, No. 1, pp. 56-61.

2 Ade P. A. R. et al. Planck 2013 results. XVI. Cosmological parameters. Astronomy \& Astrophysics. 2014, Vol. 571, pp. A16.

3 Kuo C. Y. et al. The megamaser cosmology project. V. An angular-diameter distance to NGC 6264 at 140 Mpc. The Astrophysical Journal. 2013, Vol. 767, No. 2, pp. 155-159.

4 Nojiri S. I., Odintsov S. D. Introduction to modified gravity and gravitational alternative for dark energy.International Journal of Geometric Methods in Modern Physics.2007, Vol. 4, No. 01, pp. 115-145.

5 MaederA. An alternative to the $\Lambda \mathrm{CDM}$ model: the case of scale invariance. The Astrophysical Journal. 2017,Vol. 834(2), pp. 194-200.

6 Maeder A. Scale-invariant Cosmology and CMB Temperatures as a Function of Redshifts. The Astrophysical Journal. 2017, Vol. 847, No.1, pp. 65-69.

7 Maeder A., Gueorguiev V. G. Scale-invariant dynamics of galaxies, MOND, dark matter, and the dwarf spheroidals. Monthly Notices of the Royal Astronomical Society. 2020, Vol.492, No.2, pp. 2698 - 2708.

8 Peebles P. J. E. The large-scale structure of the universe. Princeton university press. 1980, $440 \mathrm{p}$.

9 Landau L.D, Lifshitz E.M. Field theory.ElsevierScience, 2013, 218 p.

10 Nicolis G., Prigogine I. Exploring complexity an introduction. 1989, 107 p.

11 Strong M. D., Crescimanno M. Lagrange point stability for a rotating host mass binary. Physical Review D. 2020, Vol.102, No. 2, pp. 024052.

12 Zhanabaev Z.Zh. Fractal model of turbulence in the jet. Proceedings of the SB Acad.of Sci. USSR. Technical Scienceseries. 1988, Vol.4, pp. 57 - 60. [in Russian].

13 Zhanabaev Z.Zh., et al. Electrodynamic characteristics of wire dipole antennas based on fractal curves. Journal of Engineering Science and Technology. 2019, Vol.14, No. 1, pp. 305 - 320.

14 Feder J. Fractals. Springer Science \& Business Media, 2013, 183 p.

15 Chhoa J. F. An Adaptive Approach to Gibbs' Phenomenon. Master'sThessis. 2020, 111 p.

16 García-Farieta J. E., Casas-Miranda R. A. Effect of observational holes in fractal analysis of galaxy survey masks. Chaos, Solitons \& Fractals.2018, Vol. 111, pp. 128-137.

17 Karachentsev I.D., et al. A catalog of neighboring galaxies. The Astronomical Journal. 2004, Vol. 127(4), pp. 2031 .

18 Ahumada R., et al. The 16th data release of the Sloan Digital Sky Surveys: first release from the APOGEE-2 Southern Survey and full release of eBOSS Spectra. The Astrophysical Journal Supplement Series. 2020, Vol.249, No.1, pp. 3.

19 Boller T., et al. Second ROSAT all-sky survey (2RXS) source catalogue. Astronomy \& Astrophysics. 2016, Vol. 588, pp. A103.

20 Freedman W. L., et al. Final results from the Hubble Space Telescope key project to measure the Hubble constant The Astrophysical Journal. 2001, Vol. 553, No.1, pp. 47.

21 Elvis M., et al. Spectral energy distributions of type 1 active galactic nuclei in the COSMOS survey. I. The XMM-COSMOS sample. The Astrophysical Journal. 2012, Vol.759, No.1, pp. 6 - 13. 